\title{
Valid Inequalities for Mixed-Integer Programs with Fixed Charges on Sets of Variables
}

\author{
Adam N. Letchford* Georgia Souli ${ }^{\dagger}$ \\ To appear in Operations Research Letters
}

\begin{abstract}
We consider mixed 0-1 linear programs in which one is given a collection of (not necessarily disjoint) sets of variables and, for each set, a fixed charge is incurred if and only if at least one of the variables in the set takes a positive value. We derive strong valid linear inequalities for these problems, and show that they generalise and dominate a subclass of the well-known flow cover inequalities for the classical fixed-charge problem.
\end{abstract}

Keywords: polyhedral combinatorics; branch-and-cut; mixed-integer linear programming

\section{Introduction}

It is well known that a wide range of important optimisation problems can be modelled as mixed-integer linear programs (MILPs). A key ingredient of modern exact MILP algorithms is the use of strong valid linear inequalities, also known as cutting planes, to strengthen the continuous relaxation of the problem (see, e.g., $[4,5]$ ).

One strand of the literature on cutting planes is concerned with MILPs that involve fixed charges (see, e.g., [1,9-11,14,16-18,21-24,26]). A fixed charge is an additional cost that is incurred whenever a certain variable takes a positive value. The textbook way to model fixed charges is as follows. Suppose that $x_{j}$ is a continuous non-negative variable, and the fixed charge $d_{j}$ is incurred whenever $x_{j}>0$. Suppose also that we know an upper bound $u_{j}$ on the value taken by $x_{j}$ in an optimal solution. We define a new binary variable, say $y_{j}$, taking the value 1 if and only if the fixed charge

\footnotetext{
*Corresponding author. Department of Management Science, Lancaster University, Lancaster LA1 4YX, United Kingdom. E-mail: a.n.letchford@lancaster.ac.uk

${ }^{\dagger}$ STOR-i Centre for Doctoral Training, Lancaster University, Lancaster LA1 4YR, United Kingdom. E-mail: G.Souli@lancaster.ac.uk
} 
is incurred. We then add $d_{j} y_{j}$ to the cost function, and add the linear constraint $x_{j} \leq u_{j} y_{j}$.

Unfortunately, when the textbook approach is used, the continuous relaxation of the resulting MILP is often very weak. In this situation, cutting planes are essential. In particular, the so-called flow cover inequalities [21,23], and various extensions of them $[9,10,18]$, have proven to be so effective that they are now generated by default not only in commercial MILP solvers (such as CPLEX, Gurobi and Xpress), but also in open-source solvers (such as CBC and SCIP).

In this paper, we consider a more general situation, in which fixed charges are associated with sets of variables. More precisely, we suppose that we have continuous variables $x_{1}, \ldots, x_{n}$, a collection of (not necessarily disjoint) sets $S_{1}, \ldots, S_{m}$ satisfying $\bigcup_{i=1}^{m} S_{i}=\{1, \ldots, n\}$, and a fixed charge $d_{i}$ for $i=1, \ldots, m$. The idea is that, for each $i$, the fixed charge $d_{i}$ is incurred if $x_{j}>0$ for any $j \in S_{i}$. (To see how this situation could arise in practice, consider a set of products that share a common machine. If any of the products are to be manufactured on a given day, then the machine must be set up at the start of that day.)

The paper has the following structure. Section 2 is a literature review. In Section 3 , we consider the case in which the sets $S_{1}, \ldots, S_{m}$ are nested. We derive a family of valid inequalities for the associated polytope, and show that the inequalities both generalise and dominate a subclass of the flow cover inequalities. In Section 4, we extend some of our results to the general case, in which the sets can intersect in an arbitrary way. Throughout the paper, $N$ and $M$ denote $\{1, \ldots, n\}$ and $\{1, \ldots, m\}$, respectively.

\section{Literature Review}

We now review the relevant literature. In Subsection 2.1, we briefly review valid inequalities for fixed-charge problems. In Subsection 2.2, for reasons which will become clear later, we review some papers on what we call optimality cuts.

\subsection{Valid inequalities for the fixed charge polytope}

Padberg et al. [21] introduced the fixed-charge polytope, defined as the convex hull of pairs $(x, y) \in \mathbb{R}_{+}^{n} \times\{0,1\}^{n}$ satisfying

$$
\begin{aligned}
& \sum_{j=1}^{n} x_{j} \leq d \\
& x_{j} \leq u_{j} y_{j} \quad(j=1, \ldots, n),
\end{aligned}
$$

where $d$ and the $u_{j}$ are positive integers.

A set $C \subseteq N$ is called a cover if $\sum_{j \in C} u_{j}>d$. Given a cover $C$, we let $\lambda$ denote the "excess capacity" $\sum_{j \in C} u_{j}-d$, we let $u^{+}$denote $\max _{j \in C} u_{j}$, 
and we define the set $C^{*}=\left\{j \in C: u_{j}>\lambda\right\}$. Padberg et al. showed that, given a cover $C$ and a (possibly empty) set $L \subseteq N \backslash C$, the following flow cover inequality (FCI) is valid:

$$
\sum_{j \in C \cup L} x_{j} \leq d-\sum_{j \in C^{*}}\left(u_{j}-\lambda\right)\left(1-y_{j}\right)+\sum_{j \in L} \alpha_{j} y_{j}
$$

where $\alpha_{j}$ is $\max \left\{u^{+}, u_{j}\right\}-\lambda$ for $j \in L$. As a special case, when $L=\emptyset$ we obtain:

$$
\sum_{j \in C} x_{j} \leq d-\sum_{j \in C^{*}}\left(u_{j}-\lambda\right)\left(1-y_{j}\right) .
$$

We will call inequalities of this type simple FCIs.

The FCIs were extended to fixed-charge network flow problems in [23]. They have been further generalised and strengthened in various ways $[9$, 10,22,23]. A related family of inequalities, the flow pack inequalities, were studied by Atamtürk [1]. For a very different family of inequalities, derived from knapsack polytopes, see our recent paper [14].

\subsection{Optimality cuts}

By an optimality cut, we mean a linear inequality that is satisfied by all optimal MILP solutions, but may be violated by one or more sub-optimal solutions. Note that some authors expand this definition to include linear inequalities that are satisfied by at least one optimal solution. This concept appears, sometimes under different names, in many places (e.g., $[2,3,6,7$, $11-13,19])$.

Among these works, Hooker et al. [11] is itself concerned with fixedcharge problems. Consider a directed network in which, for each arc $a$, there is a continuous flow variable $x_{a}$ and a binary fixed-charge variable $y_{a}$. Consider a node $i$. Let $a$ be an arc entering node $i$, and let $\alpha, \ldots, \omega$ be the arcs leaving $i$. Suppose that $y_{a}=1$ in an optimal solution. Then $x_{a}$ must be positive, since, if that were not so, we could have made a cost saving by setting $y_{a}$ to 0 . The flow entering node $i$ via arc $a$ must then exit node $i$ via one or more of the outgoing arcs. This in turn implies that at least one of the $y$ variables associated with the outgoing arcs must take the value 1 . Thus, the inequality $y_{a} \leq y_{\alpha}+\cdots+y_{\omega}$ is an optimality cut (called a logic cut in [11]).

\section{The Nested Case}

In this section, we consider the special case in which the sets $S_{1}, \ldots, S_{m}$ are nested (that is, for all pairs $\left\{i, i^{\prime}\right\} \subset M$, we have $S_{i} \cap S_{i^{\prime}}=\emptyset, S_{i} \subset S_{i^{\prime}}$ or $S_{i^{\prime}} \subset S_{i}$ ). The section is organised as follows. In Subsection 3.1, we give some notation and terminology. In Subsection 3.2, we prove a negative 


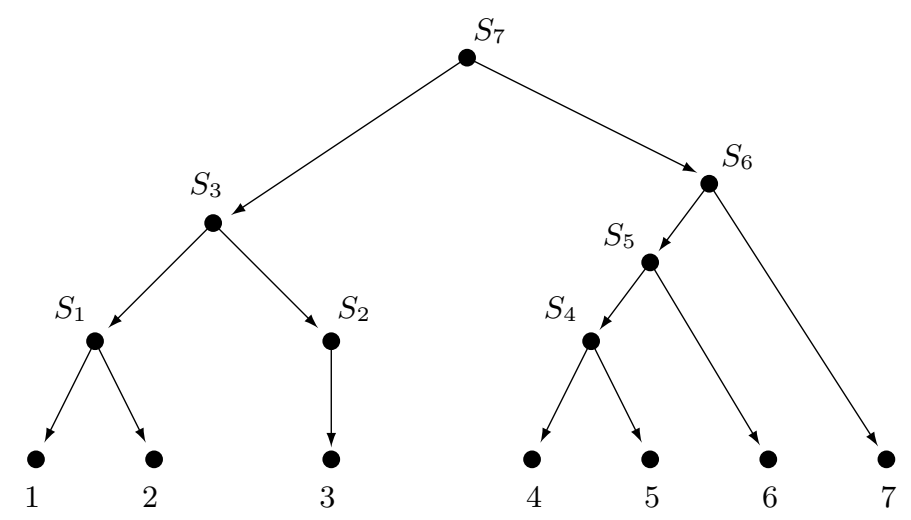

Figure 1: Visualisation of nested sets using a directed tree.

complexity result. In Subsection 3.3, we show that our problem is closely related to fixed-charge network flow (FCNF) problems. In Subsection 3.4, we present our valid inequalities. Finally, in Subsection 3.5, we consider the effect on our inequalities when optimality cuts are present.

\subsection{Notation and terminology}

From now on, we let $P$ denote the convex hull of the pairs $(x, y) \in \mathbb{R}_{+}^{n} \times$ $\{0,1\}^{m}$ satisfying

$$
\sum_{j \in S_{i}} x_{j} \leq u_{i} y_{i}(i=1, \ldots, m)
$$

Note that, in the nested case, we can assume without loss of generality that $S_{m}=N$. Indeed, if there did not exist some set $S_{i}$ that contained all the other sets, then $P$ would be the Cartesian product of simpler polytopes of the same kind.

Given $i, i^{\prime} \in M$, we will call $i$ a descendant of $i^{\prime}$ if $S_{i} \subset S_{i^{\prime}}$. If, in addition, there is no $k \in M$ such that $S_{i} \subset S_{k} \subset S_{i^{\prime}}$, we call $i$ a child of $i^{\prime}$. We let $\delta(i)$ and $\chi(i)$ denote the set of descendants and children, respectively, of $i$. We also let $\rho(i)$ denote $S_{i} \backslash \bigcup_{k \in \chi(i)} S_{k}$. (Note that at most one of $\chi(i)$ and $\rho(i)$ can be empty.) We also represent the sets $S_{1}, \ldots, S_{m}$ as a rooted directed tree, as follows. There is one node for each set $S_{i}$, and one node for each $j \in N$. There is an arc from $S_{i}$ to $S_{i^{\prime}}$ if $i^{\prime}$ is a child of $i$, and there is an arc from $S_{i}$ to $j$ if $j \in \rho(i)$.

The above notation and terminology is illustrated in the following example.

Example 1. Let $n=7$ and $m=7$, and suppose that $S_{1}=\{1,2\}$, $S_{2}=\{3\}, S_{3}=\{1,2,3\}, S_{4}=\{4,5\}, S_{5}=\{4,5,6\}, S_{6}=\{4,5,6,7\}$ and $S_{7}=\{1, \ldots, 7\}$. The corresponding tree is shown in Figure 1. One can check that, for example, 4 is a descendent of 6 and 1 is a child of 3 . One can also check that $\delta(2)=\emptyset, \delta(6)=\{4,5\}, \chi(3)=\{1,2\}, \chi(4)=\emptyset, \rho(3)=\emptyset$ and $\rho(5)=\{6\}$. 


\subsection{Complexity}

Our first result is that, even in the nested case, optimisation over $P$ is difficult.

Proposition 1 Even in the nested case, optimising a linear function over $P$ is $\mathcal{N} \mathcal{P}$-hard.

Proof. We reduce SUBSET SUM, shown to be $\mathcal{N} \mathcal{P}$-complete in [8], to the problem in question. An instance of SUBSET SUM is given by positive integers $p_{1}, \ldots, p_{k}$ and a "target" $t$. The task is to determine if there exists a subset of $\left\{p_{1}, \ldots, p_{k}\right\}$ whose sum is $t$. To convert this to our problem, set $n$ to $k$ and $m$ to $k+1$. For $i \in N$, set $S_{i}$ to $\{i\}$, set $u_{i}$ to $p_{i}$, give $x_{i}$ a profit of 1 and give $y_{i}$ a cost of $p_{i}(1-\epsilon)$, where $\epsilon$ is a small positive quantity. Finally, set $S_{m}$ to $N, u_{m}$ to $t$, and give $y_{m}$ a cost of zero. Since all $u_{i}$ values are integers, there exists an optimal solution in which $x$ is integral. Then, for any given $i \in N$, it is not worth setting $y_{i}$ to 1 unless $x_{i}$ is set to $p_{i}$, in which case we gain a profit of $\epsilon p_{i}$. So the answer to SUBSET SUM is "yes" if and only if there is a point $(x, y) \in P$ with profit $\epsilon t$. (A suitable value for $\epsilon$ is $1 / n$.)

In light of this result, we cannot expect to obtain a complete description of $P$ in terms of linear inequalities. So, we must be content with a partial linear description.

\subsection{Relation to FCNF}

Given a set of nodes $V$ and a set of $\operatorname{arcs} \mathcal{A}$, the FCNF polytope is the convex hull of the pairs $(x, y) \in \mathbb{R}_{+}^{|\mathcal{A}|} \times\{0,1\}^{|\mathcal{A}|}$, such that

$$
\begin{array}{lr}
\sum_{j \in V} x_{j i}-\sum_{j \in V} x_{i j}=b_{i} & (i \in V) \\
x_{i j} \leq u_{i j} y_{i j} & ((i, j) \in \mathcal{A}) .
\end{array}
$$

Next, we will show that, in the nested case, there is a strong connection between the polytope $P$ and FCNF problems. Consider Figure 1 once more. For $i \in M$, let $f_{i}$ denote the flow entering the node that represents $S_{i}$, and set the capacity of the corresponding arc to $u_{i}$. We also interpret the binary variable $y_{i}$ as an indicator of whether that arc is being used or not. Finally, for $j \in N$, we let node $j$ be a "sink" node, and we interpret $x_{j}$ as representing the flow entering node $j$. By construction, a triple $(x, y, f) \in$ $\mathbb{R}_{+}^{n} \times\{0,1\}^{n} \times \mathbb{R}_{+}^{n}$ represents a feasible flow if and only if $(x, y)$ satisfies (2).

This observation is formalised in the following proposition. 
Proposition 2 Suppose that $S_{1}, \ldots, S_{m}$ are nested. Let $M^{\prime}=\{i \in M$ : $\chi(i)=\emptyset\}$, and let $P^{f}$ be the convex hull of the triples $(x, y, f) \in \mathbb{R}_{+}^{n} \times$ $\{0,1\}^{m} \times \mathbb{R}_{+}^{m}$ satisfying

$$
\begin{array}{cl}
f_{i}=\sum_{j \in S_{i}} x_{j} & \left(i \in M^{\prime}\right) \\
f_{i}=\sum_{k \in \chi(i)} f_{k}+\sum_{j \in \rho(i)} x_{j} & \left(i \in M \backslash M^{\prime}\right) \\
f_{i} \leq u_{i} y_{i} & (i \in M) .
\end{array}
$$

Then (a) $P^{f}$ is an FCNF polytope of an acyclic graph, (b) $P$ is the projection of $P^{f}$ onto $(x, y)$-space, and (c) $P$ is affinely congruent to $P^{f}$.

Proof. By construction, the equations (3) and (4) enforce conservation of flow in an acyclic graph, and the constraints (5) enforce arc capacities, as well as ensuring that no flow can pass through an arc unless the arc is open. This proves claim (a). Now, by conservation of flow, we must have $f_{i}=\sum_{j \in S_{i}} x_{j}$ for all $i \in M$. Thus, if we eliminate the $f$ variables, using the equations (3) and (4), the constraints (5) reduce to (2). This proves claim (b). To see that claim (c) holds, it suffices to note that there is an invertible affine transformation which maps each extreme point of $P$ to an extreme point of $P_{f}$. This transformation consists of leaving $x$ and $y$ unchanged, and setting $f_{i}$ to $\sum_{j \in S_{i}} x_{j}$ for all $i \in M$.

This link with FCNF polytopes has an interesting consequence:

Corollary 1 Consider the following set function, which maps sets $T \subseteq N$ to $\mathbb{Z}_{+}$:

$$
\phi(T)=\max \left\{\sum_{j \in T} x_{j}: \sum_{j \in S_{i}} x_{j} \leq u_{i}(i \in M), x_{j} \geq 0(j \in N)\right\} .
$$

If the sets $S_{1}, \ldots, S_{m}$ are nested, then $\phi$ is submodular.

Proof. Wolsey ( $[26]$, Th. 4) proved the following. Let $G=(V, A)$ be a directed graph, let $u \in \mathbb{R}_{+}^{A}$ be a vector of arc capacities, and let $p \in \mathbb{R}_{+}^{A}$ be a vector of arc profits. Given any $S \subset V$, the maximum-profit flow through the arcs leaving $S$ is a submodular function of the set of arcs that are open. To apply this result to our problem, it suffices to set $S$ to the set of nodes representing $S_{1}, \ldots, S_{m}$, and set $p_{j}$ to 1 for each arc that connects a node in $S_{1}, \ldots, S_{m}$ to a node in $N$.

\subsection{The new inequalities}

From now on, we assume w.l.o.g. that, for each $i \in M$ such that $\rho(i)=\emptyset$, the condition $u_{i} \leq \sum_{k \in \chi(i)} u_{k}$ holds. (If it did not hold, then one could decrease $u_{i}$ without losing any feasible solutions.) 
Now, let $T \subseteq N$ be any set such that $\phi(T)=u_{i}$ for some $i \in M$. The following inequality is trivially valid and supporting for $P$ :

$$
\sum_{j \in T} x_{j} \leq u_{i}
$$

We will use sequential lifting $[20,25]$ to strengthen (7). More precisely, let $I=\left\{k \in M: S_{k} \cap T \neq \emptyset\right\}$, let $c$ denote $|I|$, and let $s(1), \ldots, s(c)$ be an arbitrary ordering of the elements of $I$. We will compute a valid inequality of the form

$$
\sum_{j \in T} x_{j} \leq u_{i}-\sum_{k=1}^{c} \beta_{k}\left(1-y_{s(k)}\right),
$$

where the (non-negative) coefficients $\beta_{k}$ are computed according to the given ordering.

The coefficients $\beta_{k}$ can be calculated as follows. Let $\phi(T, k)$ denote the maximum value that $\sum_{j \in T} x_{j}$ can take when $y_{s(k)}=0$. If $\phi(T, k)=u_{i}$, then $\beta_{k}=0$. Otherwise, let $\Delta(k)=\{i \in\{1, \ldots, k-1\}: s(i) \in \delta(s(k))\}$ and set

$$
\beta_{k}=u_{i}-\phi(T, k)-\sum_{\ell \in \Delta(k)} \beta_{\ell}
$$

Note that, for fixed $T$ and $k$, one can compute $\phi(T, k)$ and $\beta_{k}$ in $O(m+n)$ time. Thus, for fixed $T$, one can compute all lifting coefficients in $O(m(m+$ $n)$ ) time. The following example shows that it is possible for this procedure to yield many inequalities that define facets of $P$. It also shows that it is possible for different lifting sequences to lead to different facets.

Example 2. Suppose that $n=3, m=5, S_{i}=\{i\}$ for $i=1,2,3, S_{4}=$ $\{1,2\}, S_{5}=\{1,2,3\}, u_{i}=4$ for $i=1,2,3, u_{4}=7$ and $u_{5}=10$. Taking $T=\{1,2\}$, the inequality $(7)$ is $x_{1}+x_{2} \leq 7$. We have $I=\{1,2,4,5\}$. Lifting in the order $1,2,4,5$, we obtain $\Delta(1)=\Delta(2)=\emptyset, \Delta(3)=\{1,2\}$ and $\Delta(4)=\{1,2,3\}$. This yields $\beta_{1}=\beta_{2}=7-4=3, \beta_{3}=7-0-3-3=1$ and $\beta_{4}=7-0-3-3-1=0$. The resulting valid inequality is

$$
x_{1}+x_{2} \leq 3 y_{1}+3 y_{2}+y_{4} .
$$

One can check that different lifting orders yield seven additional inequalities:

$$
\begin{aligned}
& x_{1}+x_{2} \leq 3 y_{1}+3 y_{2}+y_{5} \\
& x_{1}+x_{2} \leq 3 y_{1}+4 y_{4} \\
& x_{1}+x_{2} \leq 3 y_{1}+4 y_{5} \\
& x_{1}+x_{2} \leq 3 y_{2}+4 y_{4} \\
& x_{1}+x_{2} \leq 3 y_{2}+4 y_{5} \\
& x_{1}+x_{2} \leq 7 y_{4} \\
& x_{1}+x_{2} \leq 7 y_{5} .
\end{aligned}
$$


One can also check (either by hand or with the help of a software package such as PANDA [15]) that all eight lifted inequalities define facets of $P$. Finally, one can check that a further sixteen facets of $P$ can be obtained by taking $T=\{1,2,3\}$ and using different lifting sequences.

We also have the following lemma:

Lemma 1 The inequalities (8) generalise and dominate the simple flow cover inequalities (1).

Proof. Let $n, d, u$ and $C$ be given, as in Subsection 2.1. To put this into our framework, set $m$ to $n+1$, and set $S_{i}$ to $\{i\}$ for $i=1, \ldots, n$. Also set $S_{m}$ and $N$ to $\{1, \ldots, n+1\}, u_{m}$ to $d$, and $T$ to $C$. We have $I=C \cup\{m\}$. Let $r$ denote $d-\sum_{j \in C^{*}}\left(u_{j}-\lambda\right)$, and note that $r>0$. One can check that, if we lift $y_{m}$ last, we obtain:

$$
\sum_{i=1}^{n} x_{j} \leq d-\sum_{j \in C^{*}}\left(u_{j}-\lambda\right)\left(1-y_{j}\right)-r\left(1-y_{m}\right) .
$$

This dominates (1).

An interesting question is whether there can exist facet-defining inequalities of the form (8) that cannot be obtained by lifting sequentially.

\subsection{Optimality cuts}

We end this section with some remarks on optimality cuts. Note that if $y_{i}$ takes the value 0 in an optimal solution, then $y_{k}$ should also take the value 0 for all $k \in \chi(i)$. Therefore, one can strengthen the LP relaxation by adding the optimality cut $y_{k} \leq y_{i}$ for all $i, k \in M$ such that $k \in \chi(i)$. Given the connection between our problem and FCNF problems mentioned in Subsection 3.2, these can be viewed as a special kind of logic cuts.

Note that, once these optimality cuts have been added, the convex hull of the feasible pairs $(x, y)$ is no longer an FCNF polytope. Nevertheless, our procedure for generating valid inequalities still applies. It turns out, however, that most lifting sequences no longer yield facets. Indeed, a necessary condition for obtaining a facet is that, for each $i \in I$ with $\delta(i) \neq \emptyset$, the descendants of $i$ are lifted before $i$ itself is lifted. The effect of this is that, for a given $T$, one of the valid inequalities dominates all of the others. This is illustrated in the following example.

Example 2 (cont.) We add the optimality cuts $y_{1} \leq y_{4}, y_{2} \leq y_{4}, y_{3} \leq y_{5}$ and $y_{4} \leq y_{5}$. Taking $T=\{1,2\}$ and lifting in the order $1,2,4,5$, as before, we obtain inequality (10). Together with the optimality cuts, this inequality dominates the other seven inequalities mentioned in Example 2. One can 
verify that it also defines a facet of the modified polytope.

In other words, the optimality cuts eliminate some of the facets of the polytope $P$. The effect is to make the polytope simpler. Note that Lemma 1 still holds for the modified polytope.

\section{The General Case}

In this last section, we consider the general case, in which the sets $S_{i}$ do not need to be nested. It turns out that this case is much more complicated, in several respects.

A first complication is that one can no longer assume that $S_{m}=N$. For example, if $n=3, m=2, S_{1}=\{1,2\}$ and $S_{2}=\{2,3\}$, then the polytope $P$ is not the Cartesian product of simpler polytopes.

A second complication is that optimising over $P$ becomes even more difficult.

Proposition 3 In the general case, optimising a linear function over $P$ is $\mathcal{N P}$-hard in the strong sense.

Proof. We reduce the maximum independent set problem, proven to be strongly $\mathcal{N} \mathcal{P}$-hard in [8], to the problem in question. Let $G=(V, E)$ be a graph with $n$ nodes and $p$ edges. Set $m$ to $n+p$. For $i=1, \ldots, n$, set $S_{i}$ to $\{i\}$, give $x_{i}$ a profit of 3 and give $y_{i}$ a cost of 2 . For $r=1, \ldots, p$, let $S_{r+i}$ contain the end-nodes of the $r$ th edge. Set all $u$ values to 1 . There exists an independent set of size $k$ in $G$ if and only if there is a solution with profit $k$.

A third complication is that one can sometimes decrease one or more $u$ values, by solving a series of LPs. This is shown in the following example.

Example 3. Let $n=3, m=7, S_{1}=\{1\}, S_{2}=\{2\}, S_{3}=\{3\}, S_{4}=\{1,2\}$, $S_{5}=\{1,3\}, S_{6}=\{2,3\}$ and $S_{7}=\{1,2,3\}$. Also, let $u_{1}=u_{2}=u_{3}=2$, $u_{4}=u_{5}=u_{6}=3$ and $u_{7}=5$. Recall the function $\phi$ from Subsection 3.3. Solving the LP (6) with $T=S_{7}$, we find that $\phi\left(S_{7}\right)=4.5$. Hence $u_{7}$ can be reduced from 5 to 4.5 .

This example also shows that, even when the original vector $u$ is integral, it is possible for $\phi(T)$ to be fractional for a given $T \subseteq N$.

A fourth complication is that the polytope $P$ is no longer an FCNF polytope in general. In fact, the function $\phi$ is no longer submodular.

Example 3 (cont.) We have $\phi\left(S_{4}\right)=\phi\left(S_{5}\right)=3, \phi\left(S_{4} \cup S_{5}\right)=\phi\left(S_{7}\right)=4.5$, and $\phi\left(S_{4} \cap S_{5}\right)=\phi\left(S_{1}\right)=2$. Since $3+3<4.5+2, \phi$ is not submodular. 
On the positive side, the function $\phi$ remains subadditive.

Proposition 4 In the general case, $\phi$ is subadditive.

Proof. Let $S, T$ be subsets of $N$. Let $x^{*}$ be the vector that maximises $\sum_{j \in S \cup T} x_{j}$ in (6). Let $x^{1}, x^{2} \in \mathcal{X}$ be defined as follows

$$
x_{j}^{1}=\left\{\begin{array}{l}
x_{j}^{*}, \text { if } j \in S \\
0, \text { if } j \notin S
\end{array} \quad x_{j}^{2}=\left\{\begin{array}{l}
0, \text { if } j \in S \\
x_{j}^{*}, \text { if } j \notin S .
\end{array}\right.\right.
$$

We have

$$
\phi(S \cup T)=\sum_{j \in S \cup T} x_{j}^{*}=\sum_{j \in S} x_{j}^{1}+\sum_{j \in T \backslash S} x_{j}^{2} \leq \sum_{j \in S} x_{j}^{1}+\sum_{j \in T} x_{j}^{2} \leq \phi(S)+\phi(T) .
$$

A fifth complication is that the closed formula (9) that we presented in Subsection 3.3 can no longer be used to compute the lifting coefficients. Moreover, even when the correct coefficients are used, the resulting inequality is no longer guaranteed to be facet-defining.

Example 3 (cont.) Suppose we take $T=\{1,2,3\}$. The inequality (7) is $x_{1}+x_{2}+x_{3} \leq 4.5$. We have $I=M$. If we lift 4,5 and 6 first, the formula (9) yields $\beta_{1}=\beta_{2}=\beta_{3}=4.5-2=2.5$, which yields the invalid inequality $x_{1}+x_{2}+x_{3} \leq-3+2.5\left(y_{4}+y_{5}+y_{6}\right)$. If we lift exactly (by solving a sequence of small mixed 0-1 LPs, as in [20,25]), then we obtain $\beta_{1}=2.5, \beta_{2}=2$ and $\beta_{3}=0$. The resulting valid inequality $x_{1}+x_{2}+x_{3} \leq 2.5 y_{4}+2 y_{5}$ does not define a facet of $P$.

In fact, we do not know whether lifting can be performed in polynomial time in the general case, and we do not have a necessary and sufficient condition for a lifted inequality (8) to define a facet. On the positive side, it turns out that the lifted inequalities still define facets of $P$ in many cases.

Example 3 (cont.) Suppose we change $u_{7}$ from 5 to 4 . Taking $T=$ $\{1,2,3\}$ again, the inequality (7) is $x_{1}+x_{2}+x_{3} \leq 4$. Lifting in the order $1,2,3,7,4,5,6$, we get

$$
x_{1}+x_{2}+x_{3} \leq y_{1}+y_{2}+y_{3}+y_{7} .
$$

Lifting in the order $1,2,5,6,3,4,7$, we get

$$
x_{1}+x_{2}+x_{3} \leq y_{1}+y_{2}+y_{5}+y_{6} .
$$

One can check that these two inequalities define facets of $P$. One can also check that, using different lifting sequences, one can obtain an additional 42 
facets.

Finally, we mention that, as in the nested case, when optimality cuts are present, most lifting sequences no longer yield facets. Interestingly, however, we can now obtain more than one facet for a given $T$.

Example 3 (cont.) Suppose that, as before, $u_{7}=4$ and $T=\{1,2,3\}$. We add the optimality cuts $y_{1} \leq y_{4}, y_{1} \leq y_{5}$ and so on. One can check that the inequalities (11) and (12) remain facet-defining, and so do the following two inequalities:

$$
\begin{aligned}
& x_{1}+x_{2}+x_{3} \leq y_{1}+y_{3}+y_{4}+y_{6} \\
& x_{1}+x_{2}+x_{3} \leq y_{2}+y_{3}+y_{4}+y_{5} .
\end{aligned}
$$

An interesting open question is whether, in the general case, one can obtain facet-defining inequalities with fractional coefficients, even if the original $u$ values are integers.

\section{Acknowledgements}

We thank Niamh Lamin for performing some preliminary polyhedral computations for us. Furthermore, the second author gratefully acknowledges financial support from the EPSRC through the STOR-i Centre for Doctoral Training under grant EP/L015692/1.

\section{References}

[1] A. Atamtürk (2001) Flow pack facets of the single node fixed-charge flow polytope. Oper. Res. Lett., 29, 107-114.

[2] E. Balas (1980) Cutting planes from conditional bounds: a new approach to set covering. Math. Program. Study, 12, 19-36.

[3] M. Bellmore \& H.D. Ratliff (1971) Set covering and involutory bases. Mgmt. Sci., 18, 194-206.

[4] M. Conforti, G. Cornuéjols \& G. Zambelli (2010) Polyhedral approaches to mixed integer linear programming. In M. Juenger et al. (eds.) 50 Years of Integer Programming, pp. 343-385. Heidelberg: Springer.

[5] W. Cook (2010) Fifty-plus years of combinatorial integer programming. In M. Juenger et al. (eds.) 50 Years of Integer Programming, pp. 387430. Heidelberg: Springer. 
[6] E. Fernández \& K. Jørnsten (1994) Partial cover and complete cover inequalities. Oper. Res. Lett., 15, 19-33.

[7] M. Fischetti, J.J.Salazar Gonzalez, \& P. Toth (1998). Solving the orienteering problem through branch-and-cut. INFORMS J. Comput., 10, 133-148.

[8] M.R. Garey \& D.S. Johnson (1979) Computers and Intractability: A Guide to the Theory of NP-Completeness. New York: Freeman.

[9] Z. Gu, G.L. Nemhauser \& M.W.P. Savelsbergh (1999) Lifted flow cover inequalities for mixed 0-1 integer programs. Math. Program., 85, 439467.

[10] Z. Gu, G.L. Nemhauser \& M.W.P. Savelsbergh (1999) Sequenceindependent lifting in mixed integer programming. J. Comb. Optim., 4, 109-129.

[11] J.N. Hooker, H. Yan, I.E. Grossmann \& R. Raman (1994) Logic cuts for processing networks with fixed charges. Comput. $\&$ Oper. Res., 21, $265-279$.

[12] K. Kaparis \& A.N. Letchford (2008) Local and global lifted cover inequalities for the multidimensional knapsack problem. Eur. J. Oper. Res., 186, 91-103.

[13] G. Lancia, F. Rinaldi \& P. Serafini (2015) Local search inequalities. Discr. Optim., 16, 76-89.

[14] A.N. Letchford \& G. Souli (2019) New valid inequalities for the fixedcharge and single-node flow polytopes. Oper. Res. Lett., 47, 353-357.

[15] S. Lörwald \& G. Reinelt (2015) PANDA: a software for polyhedral transformations. EURO J. Comput. Optim., 3, 297-308.

[16] Q. Louveaux \& L.A. Wolsey (2007) Lifting, superadditivity, mixed integer rounding and single node flow sets revisited. Ann. Oper. Res., 153, $47-77$.

[17] H. Marchand \& L.A. Wolsey (1999) The 0-1 knapsack problem with a single continuous variable. Math. Program., 85, 15-33.

[18] H. Marchand \& L.A. Wolsey (2001) Aggregation and mixed-integer rounding to solve MIPs. Oper. Res., 49, 363-371.

[19] P. Nobili \& A. Sassano (1992) A separation routine for the set covering polytope. In E. Balas, G. Cornuéjols \& R. Kannan (eds.) Proceedings of the 2nd IPCO Conference, pp. 201-219. Pittsburgh, PA: CMU Press. 
[20] M.W. Padberg (1975) A note on zero-one programming. Oper. Res., $23,833-837$.

[21] M.W. Padberg, T.J. Van Roy \& L.A. Wolsey (1985) Valid linear inequalities for fixed charge problems. Oper. Res., 33, 842-861.

[22] J.I.A. Stallaert (1997) The complementary class of generalized flow cover inequalities. Disc. Appl. Math., 77, 73-80.

[23] T.J. Van Roy \& L.A. Wolsey (1986) Valid inequalities for mixed 0-1 programs. Disc. Appl. Math., 14, 199-213.

[24] T.J. Van Roy \& L.A. Wolsey (1987) Solving mixed integer programming problems using automatic reformulation. Oper. Res., 35, 45-57.

[25] L.A. Wolsey (1976) Facets and strong valid inequalities for integer programs. Oper. Res., 24, 367-372.

[26] L.A. Wolsey (1989) Submodularity and valid inequalities in capacitated fixed charge networks. Oper. Res. Lett., 8, 119-124. 